\title{
Effect of Chitobiose and Chitotriose on Carbon Tetrachloride-Induced Acute Hepatotoxicity in Rats
}

\author{
An-Shu Chen, ${ }^{a}$ Tadao Taguchi, ${ }^{a}$ Kazuo SaKaI,${ }^{b}$ Yoshiharu Matahira, ${ }^{b}$ Min-Wei Wang, ${ }^{c}$ and \\ Ichitomo MrwA *,a \\ ${ }^{a}$ Department of Pathobiochemistry, Faculty of Pharmacy, Meijo University; Tempaku-ku, Nagoya 468-8503, Japan: \\ ${ }^{b}$ Yaizu Suisan Kagaku Industry Co.; Yaizu 425-8750, Japan: and ${ }^{c}$ Department of Pharmacology, Shenyang \\ Pharmaceutical University School of Pharmacy; Shenyang 110016, China. \\ Received May 20, 2005; accepted August 1, 2005
}

The purpose of the present study was to examine whether chitobiose and chitotriose can protect rats from $\mathrm{CCl}_{4}$-induced hepatic toxicity when given orally. We studied the effects of the 2 chitooligosaccharides given orally to rats on the acute hepatotoxicity induced by $\mathrm{CCl}_{4}$-dependent lipid peroxidation. The increase in the sum of malondialdehyde and 4-hydroxy-2-alkenals, a marker of lipid peroxidation, in both plasma and liver of $\mathrm{CCl}_{4^{-}}$ treated rats was suppressed by oral administration of chitobiose or chitotriose. The elevation in the levels of plasma aspartate transaminase and alanine transaminase activities, markers of hepatic injury, induced by $\mathbf{C C l}_{4}$ intoxication was also counteracted by oral administration of either chitooligosaccharide. The results indicate that chitobiose and chitotriose have the ability to exert a protective action against $\mathbf{C C l}_{4}$-induced acute hepatoxicity, probably by their antioxidant activity.

Key words chitobiose; chitotriose; carbon tetrachloride; antioxidant activity; hepatotoxicity

Chitosan, a polysaccharide made up of $\beta$-1,4-linked D-glucosamine residues, is produced by the deacetylation of chitin obtained from crab and prawn shells. The mixture of chitooligosaccharides, hydrolysis products of chitosan, has attracted much attention as a new biomedical material owing to its unique antibacterial, ${ }^{1,2)}$ antifungal ${ }^{3)}$ antioxidant, ${ }^{4)}$ antimutagenic, ${ }^{5)}$ and leukemia cell differentiation-inducing activities. $\left.{ }^{6}\right)$ However, these activities have been studied under in vitro, but not in vivo, conditions. In addition, the biological activities of each of the chitooligosaccharides remain to be studied.

Very recently, we reported that some chitooligosaccharides including chitobiose, chitotriose, chitotetraose, and chitopentaose showed antioxidant activity in vitro comparable to or higher than that of trolox (a water-soluble $\alpha$-tocopherol analogue) when examined with 5 assay systems. ${ }^{7)}$ In addition, we reported that chitobiose and chitotriose are appreciably absorbed from the gastrointestinal tract when given orally, whereas chitotetraose and chitopentaose are not. ${ }^{8}$ In the present study, we investigated the effects of chitobiose and chitotriose given orally to rats on the acute hepatotoxicity induced by carbon tetrachloride, which has been used as a xenobiotic to cause hepatic injury subsequent to lipid peroxidation. ${ }^{9)}$

\section{MATERIALS AND METHODS}

Materials Male Wistar strain rats (7 weeks old) were obtained from Clea Japan (Tokyo, Japan). The body weight of the rats at the time of usage ranged from 165 to $185 \mathrm{~g}$. Chitobiose and chitotriose as their hydrochlorides were purchased from Seikagaku Corp. (Tokyo, Japan). According to the HPLC analysis by the manufacturer, the purity of the 2 sugars was approximately $100 \%$. A kit (Transferase CII-test Wako) for the colorimetric assay of aspartate transaminase (AST) and alanine transaminase (ALT) activities in plasma was purchased from Wako (Osaka, Japan). A kit (Bioxytech
LPO-586) for the colorimetric assay of the sum of malondialdehyde and 4-hydroxy-2-alkenals in liver and plasma was purchased from Oxis Research (Portland, OR, U.S.A.). The LPO-586 assay is based on the reaction of a chromogenic reagent, $N$-methyl-2-phenylindole, with aldehydes.

Induction of Liver Injury Rats were fasted for $24 \mathrm{~h}$ prior to experiments, but were allowed free access to water. $\mathrm{A} \mathrm{CCl}_{4}$ solution $(50 \%, \mathrm{v} / \mathrm{v})$ in corn oil was administered intraperitoneally to rats at $1 \mathrm{ml} / \mathrm{kg}$. The rats given $\mathrm{CCl}_{4}$ were randomly divided into 3 groups. One group remained untreated, and the other groups were treated with chitobiose or chitotriose. Each chitooligosaccharide, dissolved in physiological saline, was administered orally at $300 \mathrm{mg} / \mathrm{kg}$ by gavage $30 \mathrm{~min}$ before and $3 \mathrm{~h}$ after the $\mathrm{CCl}_{4}$-poisoning. The control rats, not exposed to $\mathrm{CCl}_{4}$, were given only corn oil $(1 \mathrm{ml} / \mathrm{kg})$.

Sampling of Plasma and Liver At $6 \mathrm{~h}$ after the $\mathrm{CCl}_{4}$ treatment, blood samples were taken with heparinized syringes from a tail vein and then immediately centrifuged to obtain plasma samples. At $24 \mathrm{~h}$ after the $\mathrm{CCl}_{4}$ treatment, blood samples were collected with heparinized syringes from the abdominal aorta under anesthesia with pentobarbital; and then the livers were removed after perfusion with ice-cold physiological saline.

Assay of Transaminase Activities and Lipid Peroxidation Products AST and ALT activities in the plasma were measured by using a commercial kit. Plasma samples from $\mathrm{CCl}_{4}$-treated rats were assayed after having been diluted 20fold with $20 \mathrm{~mm}$ sodium phosphate buffer (pH 7.4). The sum of malondialdehyde and 4-hydroxy-2-alkenals in liver and plasma was measured with the Bioxytech LPO-586 kit. For the assay of the sum of malondialdehyde and 4-hydroxy-2alkenals in liver, the tissue was homogenized in 2 volumes of $20 \mathrm{~mm}$ Tris- $\mathrm{HCl}$ buffer (pH 7.4) containing $5 \mathrm{~mm}$ butylated hydroxytoluene. The homogenate was centrifuged at $9000 \times \mathbf{g}$ for $10 \mathrm{~min}$ at $4{ }^{\circ} \mathrm{C}$, after which the supernatant was analyzed for the lipid peroxidation products. 
Statistics Results were expressed as the mean \pm S.D. Statistical analysis was performed by the method of Bonferoni and Dunn.

\section{RESULTS AND DISCUSSION}

Carbon tetrachloride is metabolized by cytochrome $\mathrm{P} 450$ $2 \mathrm{E} 1$ to the trichloromethyl radical $\left(\mathrm{CCl}_{3}^{*}\right) . \mathrm{CCl}_{3}$ and its highly reactive derivative, the trichloromethylperoxyl radical $\left(\mathrm{Cl}_{3} \mathrm{COO}\right)^{*}$, are assumed to initiate free radical-mediated lipid peroxidation leading to the accumulation of lipid peroxidation products that cause hepatic injury. ${ }^{9)}$ The increase in plasma transaminase activities is known as a sign of $\mathrm{CCl}_{4}$-induced hepatotoxicity. ${ }^{10-13)}$ It is rational, therefore, to expect that antioxidative compounds can prevent $\mathrm{CCl}_{4}$ hepatotoxicity. In fact, a number of papers reported the preventive effects of various antioxidants on the accumulation of lipid peroxidation products and on the increase in plasma transaminase activities induced by $\mathrm{CCl}_{4}$ treatment. ${ }^{10-12,14)}$ In the present study, by assessing the effects of chitobiose and chitotriose on the amounts of lipid peroxidation products and the activities of plasma transaminases in $\mathrm{CCl}_{4}$-treated rats, we examined whether the 2 compounds could exert antioxidant activity when given orally.

We gave chitobiose and chitotriose to rats at $300 \mathrm{mg} / \mathrm{kg}$ by gavage $30 \mathrm{~min}$ before and $3 \mathrm{~h}$ after the injection of $\mathrm{CCl}_{4}$. Our intention of this double administration of chitooligosaccharides was to keep the plasma chitooligosaccharide concentration high as far as possible, not only at the moment of $\mathrm{CCl}_{4}$ injection but also for several hours after it; because the $T$ max values of chitobiose and chitotriose given orally at $300 \mathrm{mg} / \mathrm{kg}$ were 1.8 and $0.9 \mathrm{~h}$, respectively, and their plasma levels rapidly decreased after reaching the maximum. ${ }^{8)}$ Both chitobiose and chitotriose were reported to be scarcely metabolized and to be almost exclusively excreted into the urine when given orally, ${ }^{8}$ suggesting that the chitooligosaccharides do not affect the production of reactive radical species by metabolism of $\mathrm{CCl}_{4}$.

The values of the sum of malondialdehyde and 4-hydroxy2-alkenals, a marker of lipid peroxidation, in plasma and liver obtained $24 \mathrm{~h}$ after $\mathrm{CCl}_{4}$ treatment $(4.0 \pm 0.9 \mu \mathrm{M}$ and $43.1 \pm 2.5 \mathrm{nmol} / \mathrm{g}$ tissue, respectively) were significantly higher than the values for plasma and liver of control rats $(2.3 \pm 0.6 \mu \mathrm{M}$ and $28.5 \pm 1.1 \mathrm{nmol} / \mathrm{g}$ tissue, respectively; Fig. 1). This elevated level of lipid peroxidation products in plasma or liver caused by $\mathrm{CCl}_{4}$ intoxication was clearly depressed by oral administration of chitobiose or chitotriose (Fig. 1).

At both 6 and $24 \mathrm{~h}$ after administration of $\mathrm{CCl}_{4}$ to the rats, the aspartate transaminase and alanine transaminase activities, markers of hepatic injury, in plasma were markedly elevated compared with those in the control rats (Fig. 2). Oral administration of chitobiose or chitotriose to $\mathrm{CCl}_{4}$-treated rats significantly decreased the plasma transaminase activities at either time of sample collection (Fig. 2).

Chitobiose and chitotriose were not so much different from each other in their ameliorating effect on $\mathrm{CCl}_{4}$-induced hepatic injury as assessed from the lipid peroxidation product level and the plasma transaminase activities. This is consistent with our findings that the in vitro antioxidant activity of chitobiose was similar to or somewhat higher than that of
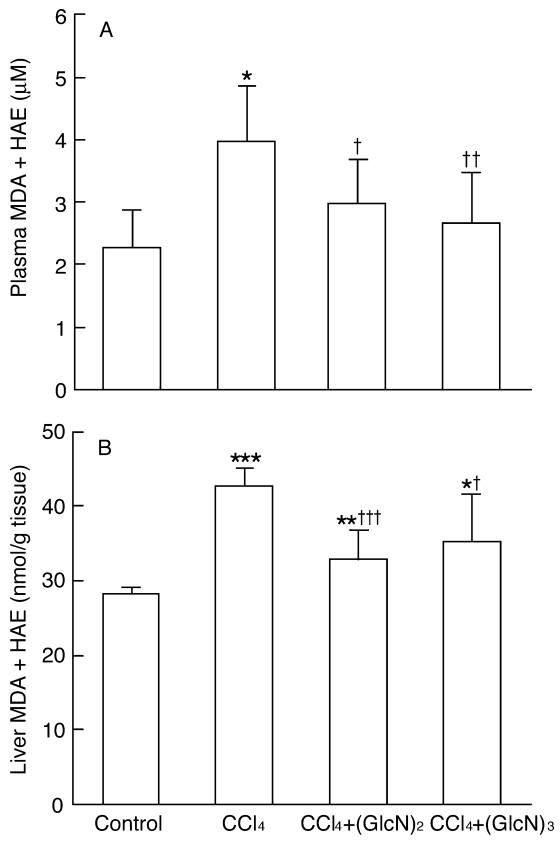

Fig. 1. Effects of Chitobiose and Chitotriose on the Sum of Malondialdehyde and 4-Hydroxy-2-alkenals in Plasma (A) and Liver (B) of $\mathrm{CCl}_{4}$-Treated Rats

Plasma and liver samples were obtained at $24 \mathrm{~h}$ after administration of $\mathrm{CCl}_{4}$ or vehicle. MDA, malondialdehyde; HAE, 4-hydroxy-2-alkenals; $(\mathrm{GlcN})_{2}$, chitobiose; $(\mathrm{GlcN})_{3}$, chitotriose. Data are means \pm S.D. of the values from 5 rats. $* p<0.05$, $* * p<0.01, * * * p<0.001$ as compared with the control group. $\dagger p<0.05, \dagger p<0.01$, ${ }_{\dagger \dagger} p<0.001$ as compared with the $\mathrm{CCl}_{4}$ group.
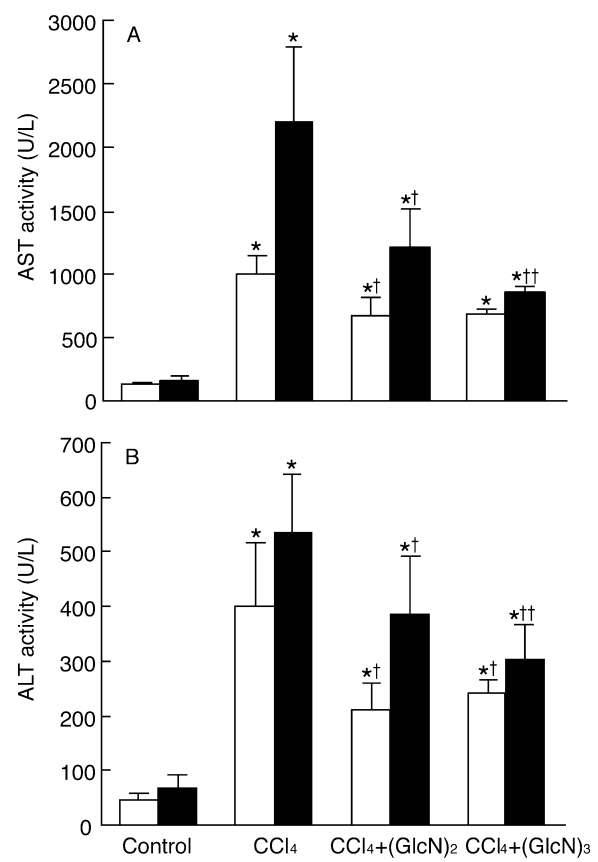

Fig. 2. Effects of Chitobiose and Chitotriose on Aspartate Transaminase (A) and Alanine Transaminase (B) Activities in Plasma of $\mathrm{CCl}_{4}$-Treated Rats

Plasma samples were obtained at 6 (open columns) and $24 \mathrm{~h}$ (closed columns) after administration of $\mathrm{CCl}_{4}$ or vehicle. AST, aspartate transaminase; ALT, alanine transaminase; $(\mathrm{GlcN})_{2}$, chitobiose; $(\mathrm{GlcN})_{3}$, chitotriose. Data are means \pm S.D. of the values from 5 rats. $* p<0.001$ as compared with the control group. $\dagger p<0.05, \dagger p<0.01$ as compared with the $\mathrm{CCl}_{4}$ group.

chitotriose when evaluated by 4 assay systems ${ }^{7}$ and that the pharmacokinetic parameters of chitobiose after oral administration at a dose of $300 \mathrm{mg} / \mathrm{kg}$ to rats were similar to those of 
chitotriose. $^{8)}$

Very recently, chitosan (MW 380000) ${ }^{14)}$ and a higher-chitooligosaccharide mixture $(3000<\mathrm{MW}<5000)^{15)}$ were reported to protect rats and mice from $\mathrm{CCl}_{4}$-induced lipid peroxidation and 2,3,7,8-tetrachlorodibenzo- $p$-dioxin-induced lipid peroxidation, respectively, when administered orally. However, there was no information on the absorbability of chitosan and the higher chitooligosaccharides in those papers. Considering our earlier findings that chitobiose and chitotriose, but not chitotetraose and chitopentaose, were absorbed from the gastrointestinal tract when given orally, ${ }^{8)}$ it is probable that chitobiose and chitotriose are produced by digestion of chitosan and higher chitooligosaccharides by intestinal bacterial flora, absorbed from the intestine, and then exert their inhibitory effect against lipid peroxidation. This view remains to be studied.

In conclusion, our results indicate that chitobiose and chitotriose given orally are able to protect rats from $\mathrm{CCl}_{4}$-induced acute hepatotoxicity, most probably by their antioxidant activity.

Acknowledgements This work was supported by a grant-in-aid (to I. M.) for the Scientific Frontier Research Project from the Ministry of Education, Culture, Sports, Science, and Technology of Japan.

\section{REFERENCES}

1) Hirano S., Nagao N., Agric. Biol. Chem., 53, 3065-3066 (1989).

2) Choi B.-K., Kim K.-Y., Yoo Y.-J., Oh S.-J., Choi J.-H., Kim C.-Y., Int. J. Antimicrobi. Agents, 18, 553-557 (2001).

3) Kendra D. F., Hadwiger L. A., Exp. Mycol., 8, 276-281 (1984).

4) Xue C., Yu G., Hirata T., Terao J., Lin H., Biosci. Biotechnol. Biochem., 62, 206-209 (1998).

5) Nam K.-S., Choi Y.-R., Shon Y.-H., Biotechnol. Lett., 23, 971-975 (2001).

6) Pae H.-O., Seo W.-G., Kim N.-Y., Oh G.-S., Kim G.-E., Kim Y.-H., Kwak H.-J., Yun Y.-G., Jun C.-D., Chung H.-T., Leuk. Res., 25, 339346 (2001).

7) Chen A.-S., Taguchi T., Sakai K., Kikuchi K., Wang M.-W., Miwa I., Biol. Pharm. Bull., 26, 1326-1330 (2003).

8) Chen A.-S., Taguchi T., Okamoto H., Danjo K., Sakai K., Matahira Y., Wang M.-W., Miwa I., Biol. Pharm. Bull., 28, 545-548 (2005).

9) Recknagel R. O., Glende E. A., Jr., Dolak J. A., Waller R. L., Pharmacol. Ther., 43, 139-154 (1989).

10) Sheweita S. A., Abd El-Gabar M., Bastawy M., Toxicology, 165, 217-224 (2001).

11) Sotelo-Félix J. I., Martinez-Fong D., Muriel P., Santillan R. L., Castillo D., Yahuaca P., J. Ethnopharmacol., 81, 145-154 (2002).

12) Ritter C., Reinke A., Andrades M., Martins M. R., Rocha J., MennaBarreto S., Quevedo J., Moreira J. C. F., Dal-Pizzol F., Crit. Care Med., 32, 2079-2084 (2004).

13) Aniya Y., Koyama T., Miyagi C., Miyahira M., Inomata C., Kinoshita S., Ichiba T., Biol. Pharm. Bull., 28, 19-23 (2005).

14) Jeon T. I., Hwang S. G., Park N. G., Jung Y. R., Shin S. I., Choi S. D., Park D. K., Toxicology, 187, 67-73 (2003).

15) Shon Y.-H., Park I.-K., Moon I.-S., Chang H. W., Park I.-K., Nam K.S., Biol. Pharm. Bull., 25, 1161-1164 (2002). 